\title{
Qatar Biobank and Qatar Genome Programs Road Map
}

\section{Althani $\mathbf{A}^{*}$}

Qatar BioBank, Qatar Genome and Biomedical Reserch Center and Department of Health Sciences, College of Arts and Sciences, Qatar University and Qatar Biobank, Qatar Foundation for Education, Science and Community

\begin{abstract}
Qatar Biobank "QBB" is a large-scale, long term medical research initiative for the population of Qatar, which will serve as a platform and driver of biomedical research to achieve benefits for Qatar's communities. Following on from the announcement of the Qatar Genome Project in 2013 by Her Highness Sheikha Moza bint Nasser, Chairperson of Qatar Foundation "QF", a National Genome Committee "NGC" is tasked with the role of driving and advising the development of the Genome Project in Qatar. To ensure the successful implementation and completion of a project of this complexity, it was important to consider carefully the organizational structure of Genome Qatar to enable success, so the decision was to establish QG project within the existing framework of the government of QBB. A road map constitutes of seven key building blocks, were identified to address the critical success factors and be managed and overseen by the Board of Trustee for both QBB and QG program. The seven building blocks are 1-Develop a National Health Information System, 2-Enhancement of the National Biobank, 3-Develop Genomics Infrastructure,4- Develop Policy Framework for genomics and precision medicine, 5-Workforce Development ,6Research and Partnership and 7- Clinical/Medical implementation. To successfully accomplish this ambitious role a road map is initiated through a pilot phase to establish the infrastructure and human capacity for 12-18 months in order to tackle problems, identify loopholes, analyze the needs and optimize systems that are needed for the entire Qatar Genome project. The aim is to sequence 1000-3000 genomes to develop a good model of practice for regulatory compliant, sample collection and storage, high quality data generation, analysis and annotation pipeline development and data warehouse establishment.
\end{abstract}

\section{Introduction}

Qatar Biobank has been created in order to enable medical research on genetic diseases that affect the local population. The Biobank aims to collect samples from the general population of Qatar as well as information on health and lifestyle factors as an initial step. The Biobank is the most ambitious national health initiative undertaken in the country with the ultimate objective of preventing, improving teh diagnosis, and treatment of genetic or complex diseases in addition to understanding how lifestyle factors affect development of disease in this population.

The baseline information from 60,000 adults will be collected by 2019 and will encompass many phenotypic characteristics, including sociodemographic information, physical measures, health and lifestyle factors, medical history, and blood measurements. The participants will be followed up for an average of 5 years for development of diabetes, cardiovascular and neurodegenrative diseases. In addition, the data from the biobank will be retrspectively linked to participants' medical records in order to elucidate any relationship between phenotypes and genotypes. The wealth of data from the Qatar Biobank will be made available to local researchers, after an approved application.

\section{Rationale for the biobank}

Complex diseases, e.g., diabetes, heart disease, etc are caused by an interplay between environmental factors and genetic predisposition. Current medical treatments for complex diseases are based on clinicla data largely from the Western population and large scale trials in the Arab region are both scant and not practical (due to the small population sizes and lack of infrastructure). In the Gulf region, to date, no large scale trials have investigated the aetiological components of complex disease. The Qatar Biobank has been set up by the Qatar Foundation and the Supreme Council of Health in collaboration with Imperial College London, as the first Qatar national population based prospective cohort study, and includes the collection of biological samples, with long-term storage of data and samples for future research.
Qatar Biobank will help identify genes and risk factors for diseases common in the homogenous Qatari population such as obesity, heart disease, diabetes and cancer. Type 2 diabetes presents a special consideration for Qatar since 17\% of the population suffer from it [1]. The Qatar Biobank will thus provide an important resource for the investigation of the aetiological factors and their interactions in the development of complex diseases. The health information and samples collected by Qatar Biobank will allow researchers to better understand diseases that affect the Qatari community and may potentially lead to a personalised medical approach towards managing this population. Qatar Biobank aims to encourage and provide as wide access as possible for local researchers to its data and samples for health-related research in the public interest.

\section{Biobank Cohort}

The potential 60,000 participants (adults ( $\geq 18$ years) who are either Qatari nationals or long-term residents (lived in Qatar for $\geq 15$ years) will be assessed between 2019 in Qatar. Blood, urine and saliva samples are being collected and stored for future research use. The assessment visit comprises of a signed consent; a self-completed questionnaire; brief healthcare professional interview; physical and functional measures; and collection of blood, urine, and saliva. Multiple aliquots of different sample fractions are stored in Qatar Biobank's laboratory, allowing for a wide range of future investigations.

*Corresponding author: Asma Althani, Vice Chair person Qatar BioBank and Chair of Qatar Genome, Department of Health Sciences, College of Arts and Sciences, Qatar University, P O Box 348, Doha, Qatar, Tel: 00974-4403-4800 Email: aaja@qu.edu.qa

Received October 29, 2015; Accepted November 12, 2015; Published November 20, 2015

Citation: Althani A (2015) Qatar Biobank and Qatar Genome Programs Road Map J Tissue Sci Eng 6: 157. doi:10.4172/2157-7552.1000157

Copyright: (c) 2015 Althani A. This is an open-access article distributed under the terms of the Creative Commons Attribution License, which permits unrestricted use, distribution, and reproduction in any medium, provided the original author and source are credited. 
Qatar Biobank will conduct a range of additional phenotyping assessments. Data are already available both from a pilot phase, completed by 1,209 participants. Over the coming months and years, further data will become available from more participants as rates of recruitment will be progressively increased in order to achieve a cohort of consented individuals broadly representative of the eligible Qatari population.

\section{Research Stemming from the Biobank}

Following initial assessment by the executive team, all research applications will be appraised and either approved or rejected by an independent Comittee. Advice is sought on any applications raising potential ethical issues from the Ethics and Governance Council. Only anonymised data are provided to researchers, who must only use it for the purposes of the approved research. Researchers must also undertake to publish their results and provide details of their methodology, derived data, and/or sample assay results for incorporation into the Qatar Biobank Database. Qatar Biobank encourages publication of results of research stemming from the Biobank.

\section{Running Qatar Biobank}

Success so far in developing and enhancing the resource has relied on public willingness to participate in the Qatar Biobank project; close engagement with volunteers, funding and governance of the Qatar Government; extensive consultation with the scientific and ethical committees; and the development of effective methodoly in association with Imperial College, UK.

A roadmap constiuting of seven key factors was identified to address the critical success factors for the project. These include:

1. Development of a National Health Information System

2. Enhancement of the National Biobank

3. Development of the Genomics Infrastructure

4. Development of Policy Framework for genomics and precision medicine

5. Workforce Development

6. Research and Partnership

7. Clinical/Medical implementation.

A key step in achieving the recruitment, characterisation, and follow-up of 60,000 participants was the creation of an executive and advisory team as well as a strong scientific and management team. This facilitated the development of a centralised infrastructure, bespoke information technology (IT) systems, and effecient approaches to collection and processing of data and samples.

Each initial recruitment visit lasts approximately 179 minutes and staff and equipment at the Biobank facilities are set up to cope with the flow of 50 pparticipants per day allowing for the recruitment visit time. Biological samples arr being processed and handled centrally. Each step of the recruitment, assessment, and sample handling process was first tested in the pilot phase and modified as necessary.

\section{Interactions with Participants}

So far participants have been recruited through public and private employers though most participants were recruited by personal recommendations of friends and family. All are requested to provide broad consent to use of their anonymised data and samples for any health-related research, to be re-contacted for further substudies, and for Qatar Biobank to access their health-related records.

Qatar Biobank keeps its participants involved through providing progress updates via its website, enabling them to continue to support the project and participate in research over the years ahead.

Participants also receive a summary of their standard measures (e.g., blood pressure, body mass index) at the end of each assessment visit and are encouraged to seek medical advice or referred if the results are outside the normal ranges. Similarly, any incidental imaging findings lead to referrals where necessary. In addition, the overall findings obtained from the pilot phase and subsequent sequencing programme will be used to identify and manage emerging public health issues in Qatar.

\section{Ethics and Governance of the Biobank}

An independent Ethics and Governance Council oversees adherence to the Ethics and Governance Framework and provides advice on the interests of research participants and the general public in relation to Qatar Biobank.

Institutional Review Board approval is obtained from the Hamad Medical Corporation Ethics Committee. The Qatar Biobank Regulations and Access Committee will advise the Board, the managing director, Qatar Foundation and Supreme Council for Health regarding the best practice and implementation of Ethics, Privacy and Access regulation as well as provide a forum for joint strategic discussion, considering actual and anticipated changes in the scope of research collaborations. It will also make recommendations for the most efficient use of QBB samples and data internally and externally.

\section{The Pilot phase}

The pilot phase ran between 2012 and 2014 and recruited 1,209 participants. Phenotypic information was collected from each participant, including socio-demographic information, health conditions, diet, lifestyle, anthropometry, body composition, bone health, cognitive function, grip strength, retinal imaging, total body dual energy X-ray absorptiometry (iDXA), and measurements of cardiovascular and respiratory function. Samples (urine, saliva and blood) were collected from each participant and stored for future use. A panel of 66 clinical biomarkers was routinely measured on fresh blood samples in all participants. The participants all underwent a 5-stage interview and physical and clinic measurement sequence which lasted approximately 179 minutes.

The results are due to be published. The pilot phase has demonstrated that recruitment and processing of data from large number of participants is logistically feasible and the data obtained will provide useful information not only on genetic basis of rare or complex diseases but also on public health issues threatening the health system in Qatar.

\section{Summary}

The key lessons learned from establishing Qatar Biobank are that this large scale project requires a focesed scientific approach, strong communication channels between the various diciplines involved; effecient processing and analysis of samples; close partnership with supporting organisations (e.g., bioinformatics support); strong overseeing scientific, legal and ethical committees, public support as well as streamlined governance and ethics infrastructure. 
The pilot phase is now complete and the sequencing of 60,000 participants is also now underway. The next steps will be to facilitate research by local scientitst and public health stakeholders in order to investigate the prevalence of certain diseases in the country as well as understand how different diseases are caused by the combination of lifestyle, environment, and genes. The aim is to eventually prevent, aid diagnosis, and treatment of identified diseases.

\section{Acknowledgement}

The author would like to thank Dr. Hanan Al Kuwari (Hamad Medical Corporation), Dr Al Marri (Hamad Medical Corporation), Dr Abdulla Al Kaabi
(Sidra Medical and Research Centre), Dr. Hadi Abderrahim (Qatar Biobank, Qatar Foundation for Education, Science, and Community), Dr Nahla Afifi (Qatar Biobank, Qatar Foundation for Education, Science, and Community), Dr Fatima Qafoud (Qatar Biobank, Qatar Foundation for Education, Science, and Community), Prof Elio Riboli (School of Public Health, Imperial College London), Prof Paul Elliott (School of Public Health, Imperial College London).

The author would like to thank Dr Sabah Allawati (Medcommz Ltd) for editorial assistance.

\section{Reference}

1. Christos PJ, Chemaitelly H, Abu-Raddad LJ, Ali Zirie M, Deleu D, et al. (2014) Prevention of type II diabetes mellitus in Qatar: Who is at risk? Qatar Medical Journal 2: 70-81. 